\title{
The Fatigue Fracture Criterion Based on the Latent Energy Approach
}

\author{
Alexander R. Arutyunyan, Robert A. Arutyunyan \\ Faculty of Mathematics and Mechanics, Sankt-Petersburg State University, Sankt-Petersburg, Russia \\ E-mail: Robert.Arutyunyan@paloma.spbu.ru \\ Received November 24, 2009; revised February 8, 2010; accepted February 12, 2010
}

\begin{abstract}
The extensive literature on the fatigue problem, published for more than one hundred years, is reviewed by the known scientists [1,2]. As it follows from these investigations, the fundamental amount of failures in engineering practice connected with the fatigue fractures of materials and structure elements. The fatigue problem is complicated one and it is not solved yet. So the theoretical and experimental investigations of this problem will be continued. In our paper the energy approach to formulate the fatigue strength criterion is proposed. The criterion is based on the conception of the latent energy [3-7]. This conception was not applied previously to the fatigue problem. The latent energy is consumed to generate the irreversible deformation and to damage and fracture of metallic materials. So the fatigue fracture criterion can be formulated using the results of latent energy measurements in the macro experiments. This is most impotent advantage of the proposed approach. The logistic function is used to describe the dependence of latent energy from the value of irreversible deformation. It is assumed that the cyclic strength of metals is defined by the latent energy, stored in specimen, when it is reached the critical value in accordance with the logistic curve in a saturation zone. This proposal is used to formulate the fatigue strength criterion. The functions and parameters of received criterion are concretized and comparisons with experimental results for axial cyclic tension for sheet aluminum alloy specimens are given.
\end{abstract}

Keywords: Fatigue Fracture Criterion, Energy Approach, Latent Energy, Heat Energy, Logistics Function, Damage Parameter

\section{Introduction}

The energy methods are the most fundamental approach for receiving the deformation and fracture laws for materials and structures. It is well known that the fracture mechanics as a science was originated by a Griffith's energy concept. In this paper the results of recent experimental investigations on latent energy stored in metallic materials during the plastic deformation are used to formulate the fatigue fracture criterion for metallic materials.

In the process of plastic deformation the significant changes in metallic materials due to increasing of the interior energy are occurred. During the plastic deformation heat is emitted so the deformation energy is transformed into the heat. But not the whole work is emitted to heat. A part of it called the latent energy of deformation [3-7] is transformed to the potential energy of lattice distortion. The latent energy in average constitutes $10-15 \%$ of full deformation energy.
The methods of measuring the latent energy are reduced to measure, with a sufficient accuracy, the deformation energy and emitted heat. In this case the energy balance is defined by the first low of thermodynamics, which can be written as $U=E-Q$, where $U$ is interior (latent) energy, $E$ is the deformation energy and $Q$ is the heat.

The results of experiments on latent energy carried out in recent years [8-10] show that the relation of the latent energy or the stored energy is expressed in the form of logistics function. Our experiments [11] conducted on cyclic bending of specimens made of construction materials show that the relations of different physical and mechanical characteristics from number of cycles has two precisely expressed regions. Similarly to the logistics function these relations have the precisely expressed point of inflection. These results are the additional confirmation for the introduction of the logistics function when formulating the fatigue strength criterion. We as- 
sume that metals cyclic strength is defined by the value of latent energy, when it is reached the critical value.

\section{Damage Parameter, Based on the Relative Changes of Latent Energy}

Let's introduce the parameter $\gamma$, which characterizes the relative changes of latent energy $\gamma=\frac{W}{W_{*}}$, where $W$ is the current and $W_{*}$ is the limiting values of latent energy, $\gamma_{0} \leq \gamma \leq \gamma_{*}$, where $\gamma_{0}, \gamma_{*}$ are initial and limiting values of parameter $\gamma$, consequently, $\gamma_{0}<\gamma_{*} \leq 1$.

It is well known that the logistics function describes the different processes which lead to saturation [12-14], and it is the solution of the following kinetic equation

$$
\frac{d \gamma}{d \varepsilon}=A \gamma\left(\gamma_{*}-\gamma\right)
$$

where $A$ and $\gamma_{*}$ are constants. In the common case $A$ and $\gamma_{*}$ can be considered as functions of stress $A=A(\sigma), \gamma_{*}=\gamma_{*}(\sigma)$.

Solving Equation (1) with the initial conditions $\varepsilon=0$, $\gamma=\gamma_{0}$, we will have the following relation, expressed in the form of logistic function

$$
\gamma=\frac{\gamma_{*}}{1+\left(\frac{\gamma_{*}}{\gamma_{0}}-1\right) \times e^{-A \varepsilon \gamma_{*}}}
$$

The theoretical curve (2) of latent energy accumulation is shown in Figure 1. The results of experiments on specimens made of aluminum alloy 2024-T351 [10] are shown in this figure by points. In calculations the following values of constants were used: $A=26,852$, $\gamma_{0}=0,02$ and $\gamma_{*}=0,94$. It is seen that the experimental points of latent energy accumulation are well described by the logistic function (2).

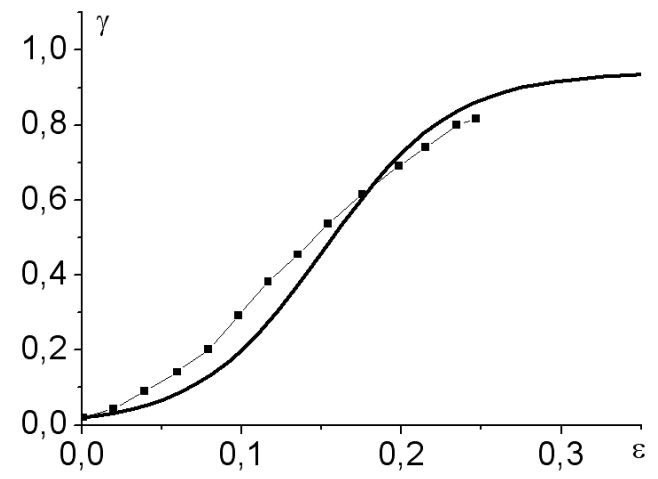

Figure 1. Solid line - the theoretical curve (2), pointsexperimental results $[10]$.
Introducing the fracture condition in the form $\gamma=a \times \gamma_{*}$, where $a$ is constant, $0,5 \leq a \leq 1$, from (2) we can receive the following relation

$$
\varepsilon=\frac{1}{A \gamma_{*}} \ln \left(\frac{a}{1-a}\left(\frac{\gamma_{*}}{\gamma_{0}}-1\right)\right)
$$

\section{The Fatigue Fracture Criterion}

It is known [15,16], that in process of cyclic loadings, in particular in cyclic tension regime, the accumulation of plastic deformation from cycle to cycle is occurred. The character of the plastic deformation accumulation is similar to creep curves. So to describe such curves the power Norton's law can be used. This law, expressed through the number of cycles, can be written in the following form

$$
\frac{d \varepsilon}{d N}=B \sigma^{m}
$$

We will assume that the stress in one cycle is constant and is equal to the amplitude of stress $\sigma$. Using the initial condition $N=0, \varepsilon=0$ the solution of Equation (4) can be found as

$$
\varepsilon=B \sigma^{m} N
$$

From the relations (3) and (5) follows the fatigue fracture criterion

$$
N=\frac{1}{A B \sigma^{m} \gamma_{*}} \ln \left(\frac{a}{1-a}\left(\frac{\gamma_{*}}{\gamma_{0}}-1\right)\right)
$$

Experiments show [17] that the latent energy decreases when stress is increases. So this relation can be expressed by the decreasing power or exponential functions of stress

$$
\begin{gathered}
\gamma_{*}=(1+c \times \sigma)^{-\beta} \\
\gamma_{*}=e^{-\alpha \sigma}
\end{gathered}
$$

where $\alpha, \beta, c$ are constants.

In view (7) and (8) the fracture criterion (6) will be written as

$$
\begin{array}{r}
N=\frac{(1+c \sigma)^{\beta}}{A B \sigma^{m}} \ln \left(\frac{a}{1-a}\left(\frac{(1+c \sigma)^{-\beta}}{\gamma_{0}}-1\right)\right) \\
N=\frac{1}{A B \sigma^{m} \times e^{-\alpha \sigma}} \ln \left(\frac{a}{1-a}\left(\frac{e^{-\alpha \sigma}}{\gamma_{0}}-1\right)\right)
\end{array}
$$

\section{Comparison of the Fatigue Fracture Criterions with the Experimental Results}

The theoretical fatigue curves (9) and (10) were compared 


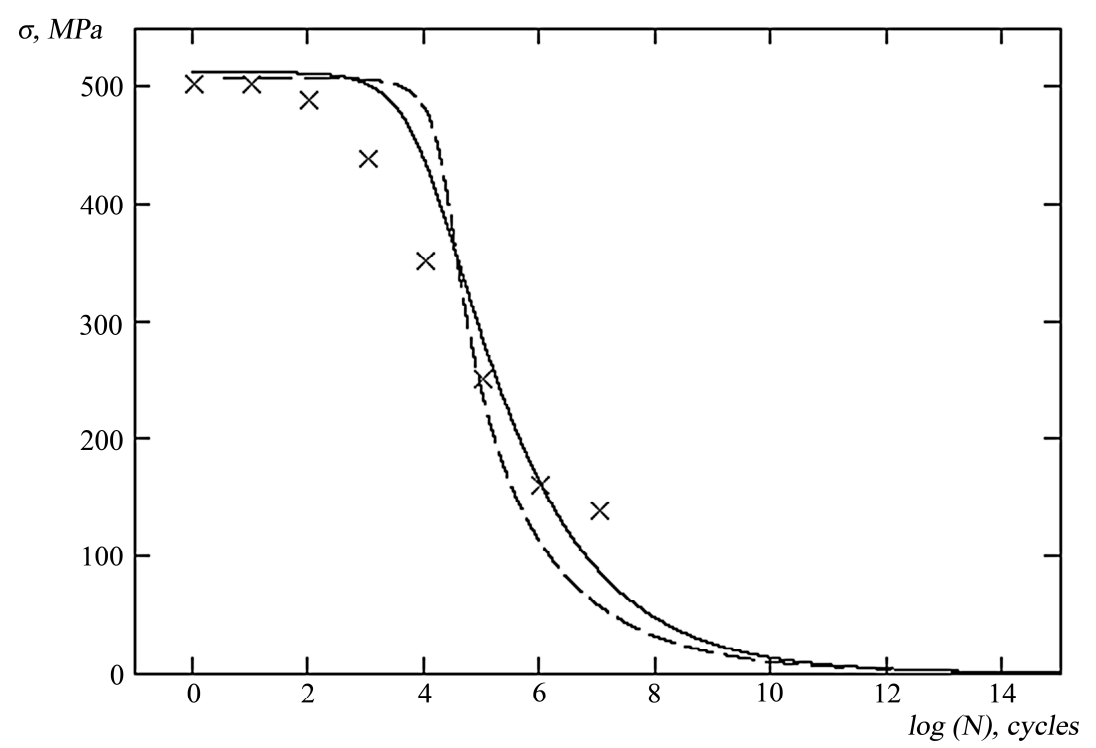

Figure 2. The theoretical fatigue curves (9) - solid line and (10) - dash line. Cross points - experimental results [15].

with the experimental results received in axial cyclic tensile tests on sheet specimens made of aluminum alloy 2024-T3 [15]. The mechanical properties of this alloy are more similar to the consequent properties of aluminum alloy used in paper [10].

The theoretical fatigue curves (9) and (10) are shown in Figure 2 by solid and dash lines consequently. The following values of constants were accepted:

$$
\begin{gathered}
A=26,852, \quad m=4, \quad B=3 \times 10^{-15}[\mathrm{MPa}]^{-4}[\text { cycles }]^{-1}, \\
a=0,9, \quad \alpha=7,5 \times 10^{-3}[\mathrm{MPa}]^{-1}, \quad c=[\mathrm{MPa}]^{-1}, \\
\beta=0,61 .
\end{gathered}
$$

The experimental results are marked by cross points. It is seen that these curves are well describe the experimental points on the whole range of fatigue curves.

\section{Conclusions}

The damage parameter defined as the relative changes of latent energy is introduced. It is shown, that this parameter is governed by the differential equation with the solution in the form of the logistics function, capable to describe different processes leading to saturation.

The functions and parameters of received criterion are concretized and comparisons with the experimental results for axial cyclic tension for sheet aluminum alloy specimens are given. A good agreement of theoretical and experimental results is received.

\section{Acknowledgements}

Financial support of the Russian Foundation for Basic
Research (Grant N 09-01-00513) is gratefully acknowledged.

\section{References}

[1] S. S. Manson, "Fatigue: A Complex Subject-Some Simple Approximations," Experience Mechanics, Vol. 7, 1965, pp. 193-225.

[2] J. Schijve, "Fatigue of Structures and Materials in the 20th Century and the State of the Art," International Journal of Fatigue, Vol. 25, 2003, pp. 679-702.

[3] G. I. Taylor and W.S. Farren, "The Heat Developed during Plastic Extension of Metals," Proceedings of the Royal Society A, London, Vol. 107, 1925, pp. 422-451.

[4] G. I. Taylor and H. Qunnney, "The Latent Energy Remaining in a Metal after Cold Working," Proceedings of the Royal Society A, London, Vol. 143, 1934, pp. 307-326.

[5] M. A. Bolshanina and V. E. Panin, "The Latent Energy of Deformation," Issledovanie po fizike tverdogo tela, Academy of Science of USSR, Moscow, 1957, pp. 193-234.

[6] O. W. Dillon, "The Heat Generated during Torsional Oscillations of Copper Tubes," International Journal of Solids and Structures, Vol. 2, 1966, pp. 181-204.

[7] M. B. Bever, D. L. Holt and A. L. Titchener, "The Stored Energy of Cold Work," Progress in Materials Science, Vol. 17, 1973, pp. 5-177.

[8] W. Oliferuk, M. Maj and B. Raniecki, "Experimental Analysis of Energy Storage Rate Components during Tensile Deformation of Polycrystals," Materials Science and Engineering A, Vol. 374, 2004, pp. 77-81.

[9] O. Plechov, N. Saintier and O. Naimark, "Experimental Investigation of the Processes of Energy Storage and Dissipation in Iron during the Elastic Plastic Deformation," 
Journal of Technical Physics, Vol. 9, 2007, pp. 135-137.

[10] P. Rosakis, A. J. Rosakis, G. Ravichandran and J. Hodowany, "A Thermodynamic Internal Variable Model for the Partition of Plastic Work into Heat and Stored Energy in Metals," Journal of the Mechanics and Physics of Solids, Vol. 48, 2000, pp. 581-607.

[11] A. R. Arutyunyan, B. A. Zimin, Y. V. Sud'enkov, "The Application of Optics-Spectroscopy Method to Investigate Fatigue of Construction Materials," Proceedings of International Conference on Topical Problems of Continuum Mechanics, Erevan, 2007, pp. 63-68.

[12] V. I. Arnold, "Ordinary Differential Equations," Nauka, Moscow, 1971.

[13] R. A. Arutyunyan, "The Problem of Deformation Aging and Prolonged Fracture in Material Science," S.-Petersburg University Press, Sankt-Petersburg, 2004.

[14] A. N. Orlov, "The Long-Time Strength and Physics of Fracture," Trudy TsKTI, Vol. 230, 1986, pp. 42-46.

[15] P. G. Forrest, "Fatigue of Metals," Mashinostroenie, Moscow, 1968.

[16] V. T. Troschenko, "Fatigue and Nonelasticityof Metals," Naukova Dumka, Kiev, 1971.

[17] O. Plechov, O. Naimark, R. Valiev, I. Semenova, N. Saintier and T. Palin-Luc, "Experimental Investigation of Anomalous Energy Absorption in Nanocrystal Titan Under the Cyclic Loading," Letters in Journal of Technical physics, Vol. 34, No. 13, 2008, pp. 33-40. 